\title{
5 Hypotheses for the experiment
}

Previous research has shown that blind listeners outperform sighted listeners in a variety of auditory tasks, especially when the tasks involve higher cognitive functions (see section 2.2) and when a physical strategy of encoding (Juurmaa 1967; Röder and Rösler 2003) is used. Unfamiliar speaker identification is also a complex auditory task in which acoustic feature analysis (i.e. physical encoding) plays a key role (cf. Kreiman and Sidtis 2011, p. 184), thus one could assume a superiority of blind over sighted listeners in this task. Previous research in this area, however, yielded conflicting results (see section 2.5.).

Since the present project is the first in which blind and sighted listeners' incidental long-term memory for speaker identification is tested with the help of open test ${ }^{14}$ voice lineups, the experiment is exploratory in nature and no specific hypotheses can be formulated with regard to the performance of blind compared to sighted listeners. It remains to be shown whether the superiority of blind over sighted listeners in other auditory abilities also gives the blind listeners an advantage in the speaker identification experiment of the present thesis. If so, it also remains to be shown whether the latter only holds true for congenitally blind listeners or also individuals who became blind later in life.

With regard to different time intervals between the first exposure to the target voice and the speaker identification test (voice lineup), it is hypothesized that listeners' speaker identification performance will get poorer over time. Additionally, it is expected that the results will be better when the speech material utilized for the experiment is presented in studio quality as compared to speech material in cell phone or whispered quality since degraded speech material contains fewer speaker-specific cues which listeners can use for identification. Musically trained listeners are expected to perform better than listeners without musical training and male listeners are expected to perform equally well as female listeners.

14 Open test voice lineups = voice lineups in which the target voice may or may not be present (cf. Elaad et al. 1998, p. 86). 